\title{
Genomic alterations on 8p21-p23 are the most frequent genetic events in stage I squamous cell carcinoma of the lung
}

\author{
JIUN KANG \\ Department of Biomedical Laboratory Science, Korea Nazarene University, Cheonan 330-718, Republic of Korea
}

Received April 23, 2014; Accepted October 31, 2014

DOI: 10.3892/etm.2014.2123

\begin{abstract}
Genetic alterations in the early stages of cancer have a close correlation with tumor initiation and potentially activate downstream pathways implicated in tumor progression; however, the method of initiation in sporadic neoplasias is largely unknown. In this study, whole-genome microarray-comparative genomic hybridization was performed to identify the early genetic alterations that define the prognosis of patients with stage I squamous cell carcinoma (SCC) of the lung. The most striking finding was the high frequency of copy number losses and hemizygous deletions on chromosome 8p, which occurred in $94.7 \%$ (18/19) and $63.2 \%$ $(12 / 19)$ of the cases, respectively, with a delineated minimal common region of 8p21.1-p23.3. More specifically, three loci of homozygous deletions at 8p23.1 were noted in $21.1 \%(4 / 19)$ of the cases. This region contains the following possible target genes, which have previously not been implicated to play a pathogenic role in stage I SCCs: MSRA, MFHAS1, CLDN23, DEFB106A, DEFB105A, LOC441316, FAM90A7P and $L O C 441318$. These findings indicate that genetic alterations on chromosome $8 \mathrm{p}$ may be the first step in the initiation of genomic instability in early SCCs, and the newly identified genes in the 8p23.1 chromosomal region might be of interest for the study of the pathophysiology of stage I SCC, as potential targets for therapeutic measures.
\end{abstract}

\section{Introduction}

Non-small cell lung cancer (NSCLC) has a high global cancer-related mortality rate (1). Two main types of NSCLC, namely adenocarcinoma (AC) and squamous cell carcinoma (SCC), are the most common subtypes. SCC of the lung is characterized by a complex pattern of cytogenetic and molecular

Correspondence to: Professor Jiun Kang, Department of Biomedical Laboratory Science, Korea Nazarene University, 456 Ssangyong-dong, Seobuk-gu, Cheonan, Chungnam 330-718, Republic of Korea E-mail: jukang@kornu.ac.kr

Key words: squamous cell carcinoma of the lung, copy number loss, homozygous deletion, tumor suppressor genes, microarray-comparative genomic hybridization genetic changes, and chromosomal aberrations are a hallmark of cancer cells, occurring at a high prevalence in SCC. Although a number of studies have been performed to evaluate genetic events associated with the development and progression of SCC $(2,3)$, the molecular mechanism remains to be uncovered, and the identification of predictive markers is crucial.

Genetic alterations in the early stages of cancer have a close correlation with tumor initiation, and potentially activate downstream pathways that are implicated in tumor progression. With the recent advances of computed tomographic technology, the number of patients diagnosed with stage I lung SCC has been increasing (3). Although stage I SCCs are thought to be early-stage diseases and are treated primarily by surgery without adjuvant therapy, a considerable fraction of the patients with such SCCs have shown unfavorable outcomes following surgical treatment. Therefore, to improve the prognosis of those patients, it is necessary to identify suitable markers to select patients with poor prognosis, who would benefit from the use of adjuvant therapy following surgery (4). In particular, the identification of the genes that are altered during cancer initiation and progression can be valuable as therapeutic targets or prognostic indicators.

Studies have previously been performed to evaluate the genetic events associated with the initiation and progression of SCCs $(5,6)$. However, little is known about the specific underlying genes that affect tumorigenesis in early stage SCCs. Furthermore, the genomic markers that predict aggressive clinical behavior of SCC remain to be identified. Therefore, in the present study, high-resolution array-comparative genomic hybridization $(\mathrm{CGH})$ was conducted to identify early genetic alterations that define the prognosis of patients with stage I lung SCC.

\section{Materials and methods}

Tumor samples and DNA extraction. A total of 19 stage I SCCs from lung patients undergoing surgery as primary treatment, without previous radiation or chemotherapy, were analyzed. The demographic and pathological data, including age, gender and tumor stage, were obtained by a review of the medical records. All the patients were classified according to the World Health Organization classification histologic typing of lung carcinomas (7). This study has been reviewed and approved by the Institutional Review Board of the Chungnam National University Hospital (Daejeon, Korea); informed consent was 
obtained from the patients for the use of their tumors in the current study.

Array-CGH experiment. The MacArray ${ }^{\mathrm{TM}}$ Karyo 4000 chips (Macrogen, Seoul, Korea) used in this study consisted of 4,046 human bacterial artificial chromosomes (BACs), which were applied in duplicate, and had a resolution of $1 \mathrm{Mbp}$ (http:// www.macrogen.co.kr) (8-11). Array-CGH was performed as described previously (12). Briefly, hybridizations were performed in a sealed chamber for $48 \mathrm{~h}$ at $37^{\circ} \mathrm{C}$. Following the hybridization, array slides were scanned on a GenePix 4200A two-color fluorescent scanner (Molecular Devices Corporation, Sunnyvale, CA, USA) and quantification was performed using GenePix 4200A software (Molecular Devices Corporation). After scanning, the fluorescent intensities of the red and green channels were saved as two TIFF image files and the background was subtracted. Locally weighted scatterplot smoothing (LOWESS) normalization was applied to adjust for effects due to variation in the intensities between the red and green dyes. The breakpoint detection and status assignment of genomic regions was performed using a Gaussian model-based approach (GLAD) (13). A low-level copy number gain was defined as a $\log _{2}$ ratio $>0.25$, and a copy number loss was defined as a $\log _{2}$ ratio $<-0.25$. This threshold value was defined empirically as a value 3 -fold that of the standard deviation calculated from hybridization experiments of 30 normal males to normal females.

Statistical analysis for array-CGH. The Fisher exact test utilized two categories, normal and abnormal (loss and gain), with the null hypothesis that the relative proportions of the two categories would be the same in different groups. A multiple testing correction [Benjamini-Hochberg false discovery rate (FDR)] was applied to correct for the high number of false positive calls. $\mathrm{R}$ package version 2.2.1 of the Bioconductor Project (http://www.bioconductor.org) was used for detection of the frequency of gain or loss and statistical analysis.

\section{Results}

Whole genome array analysis of stage I lung SCC cases. A whole genome array-CGH was conducted to investigate DNA copy number alterations, and to identify new candidate genes in 19 patients with stage I SCC. Frequent gains were seen on chromosome arms 5p, 7p, 7q, 8q, 11q and 16p ( $>40 \%$ of patients), and frequent losses on $5 \mathrm{q}, 8 \mathrm{p}, 9 \mathrm{q}, 13 \mathrm{q}$, $14 q, 15 q, 17 p$ and $22 q$ ( $>40 \%$ of patients; Fig. 1A). For the first step of the analysis, a decision was made to focus on chromosome $8 \mathrm{p}$, the most frequently lost $(94.7 \%, 18 / 19)$ and hemizygously deleted $(63.2 \%, 12 / 19)$ region in stage I SCCs. More specifically, three loci of homozygous deletions (HDs) were displayed in $21.1 \%$ (4/19) of the cases. Due to the high frequency of chromosomal imbalances observed, it was hypothesized that deletions at $8 \mathrm{p}$ must be important and early genetic events in the pathogenesis of lung SCC, and that $8 \mathrm{p}$ may harbor tumor suppressor genes (TSGs) important for early SCCs. The minimal common region of chromosome $8 \mathrm{p}$ was identified to be located between BAC19_P21 and BAC181_E17 by genome-wide array-CGH.
Chromosomal alterations of 8p21.1-p23.3 are the most common genetic alterations in stage I lung SCC cases. The most frequent regions of copy number alterations in these cases of SCC were defined more clearly, and narrowed down to 8p21.1-p23.3. A more detailed analysis of chromosome $8 \mathrm{p}$ identified three distinct regions of alterations across the chromosome. The minimal common region identified by array- $\mathrm{CGH}$ was located between BAC117_I18 and BAC250_C10. The delineation of 8p21.1-p23.3 chromosomal regions and possible target genes of the SCC cases are shown in Table I.

The first candidate locus spanned 82.0-93.2 kb in the 8p21.1-p21.3 region. According to the information archived in the human genome databases (http:/genome.ucsc.edu/), it is flanked by the BAC clones BAC117_I18 and BAC176_L07, and contains 63 possible target genes (1.8 Mb segment). Notably, a high-frequency of copy number losses $\left(-0.25>\log _{2}\right.$ ratio) and hemizygous deletions $\left(-0.5<\log _{2}\right.$ ratio $\left.<-1\right)$ at these region were detected in $47.4 \%$ (9/19) and $10.5 \%$ (2/19) of the cases, respectively.

The second locus spanned 88.6-114.6 kb in the $8 \mathrm{p} 22$ region, and demonstrated a high frequency of copy number losses in 7 of 19 cases $(36.8 \%)$. This locus comprised the putative TSGs of deleted in liver cancer 1 ( $D L C 1)$, tumor suppressor candidate 3 (TUSC3) and microtubule-associated tumor suppressor 1 (MTUS1). Furthermore, the following possible target genes were detected that have not previously been considered to play a pathogenic role in SCCs: NAT2, LOC392206, LOC137012, NAT1 and PDGFRL. A high frequency of hemizygous deletions $\left(-0.5<\log _{2}\right.$ ratio $\left.<11\right)$ in this region was also noted in $26.3 \%$ (5/19) of the cases.

The third locus was located distally in the 8p23.1-p23.3 region (87.5-104.7 kb). Notably, a high-frequency of copy number losses and hemizygous deletions at this region was detected in $89.5 \%(17 / 19)$ and $52.6 \%(10 / 19)$ of the SCCs, respectively. More specifically, three $\mathrm{HD}\left(-1<\log _{2}\right.$ ratio) loci at the 8 p23.1 region were noted in $21.1 \%$ (4/19) of the cases. The first locus contained clones covering a region of $\sim 112.8 \mathrm{~kb}$, and comprised the CLDN23 and MFHAS1 genes, occurring in $15.8 \%(3 / 19)$ of the cases. The second locus spanning $\sim 89.6 \mathrm{~kb}$ was found to contain the MSRA gene, placing it at the highest level of HD $(21.1 \%, 4 / 19)$. The third locus spanned $\sim 105.3 \mathrm{~kb}$, which contains the DEFB106A, DEFB105A, FAM90A18P, FAM90A9P, FAM90A10P and LOC441329 genes, also showing a high-frequency of HDs in the cases $(21.1 \%, 4 / 19)$. The median span of the HDs was 7.5 Mb (range, 105.3-112.8 kb), and all HDs were located between BAC90_ M06 and BAC234_K05. Representative genome profiles of HDs at the 8p23.1 region are presented in Fig. 1. Whole genome profiles are shown in the upper portion (Fig. 1A), and an individual profile of chromosome 8 , including HDs at the 8p23.1 region, is presented in more detail below (Fig. 1B). An example of an individual profile showing HDs in the 8q23.1 region is presented in Fig. 2, and a schematic presentation of the cytogenetic bands, as well as map positions, is provided underneath.

\section{Discussion}

In this study, whole-genome array-CGH showed that stage I lung SCCs display non-random patterns of co-occurring gains 
A

Chromosome : ALL
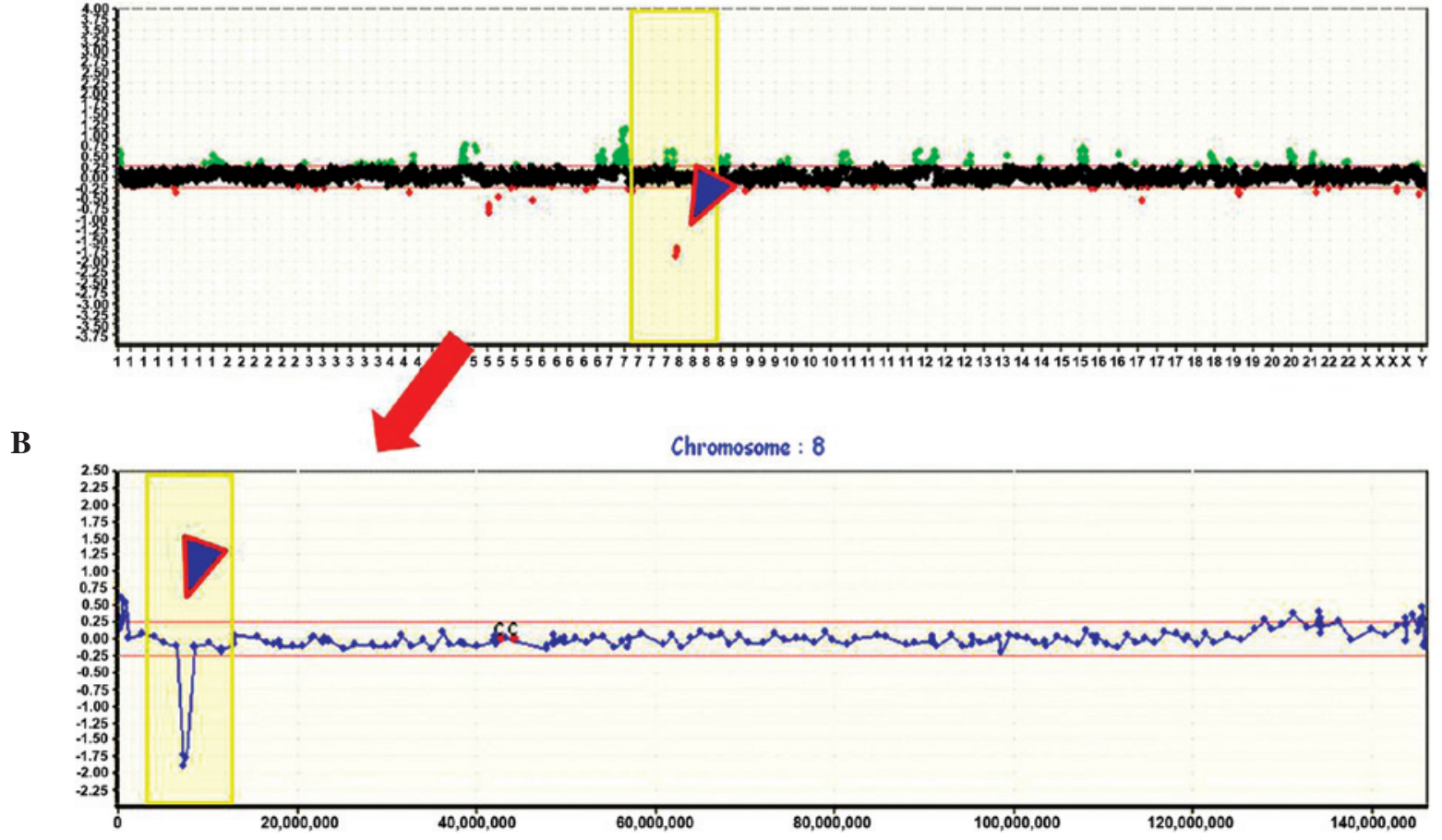

Figure 1. Examples of microarray-comparative genomic hybridization results from a patient sample (Tumor 2). (A) A $\log _{2}$ ratio $>0.25$ represents a genomic copy number gain, and a $\log _{2}$ ratio $<-0.25$ represents a genomic copy number loss. Clones are ordered from chromosome $1 \mathrm{p}$ to $22 \mathrm{q}$. For the profiles, the $\mathrm{x}$-axis represents the mapped position of the corresponding clone, and the intensity ratios are assigned to the $\mathrm{y}$-axis. The homozygous deletions (HDs) at 8p23.1 are highlighted in yellow. (B) Genomic profiles of chromosome 8 from a patient sample (Tumor 2). The vertical lines indicate a ratio of $<-1$ in this bacterial artificial chromosome (BAC) clone, suggesting HD regions at 8p23.1. The HDs at 8p23.1 are highlighted in yellow.

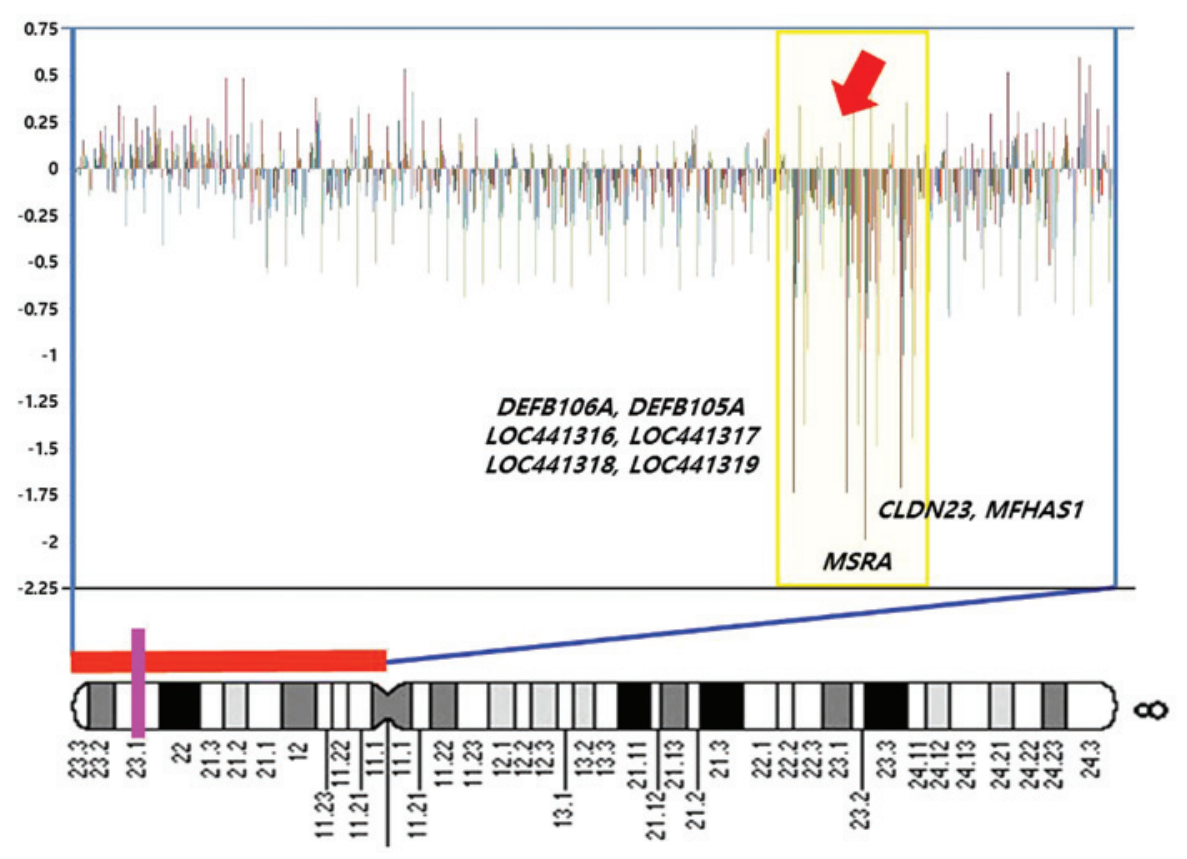

Figure 2. A diagram showing weighted frequencies (\%) of squamous cell carcinoma cases on the short arm of chromosome 8 . In the profiles, the y-axis represents the mapped position of the corresponding clone, and the intensity ratios are assigned to the $\mathrm{x}$-axis. Cytobands are shown at the bottom of the ideogram. Vertical lines indicate the lowest locus of chromosome 8 in the bacterial artificial chromosome (BAC) clone containing the MSRA, MFHAS1, CLDN23, DEFB106A, DEFB105A, LOC441316, LOC441317 (FAM90A7P) and LOC441318 genes. The homozygous deletions (HDs) at 8p23.1 are highlighted in yellow. $\log _{2}$ ratio <-1 in this BAC clone, suggesting that homozygous deletions occurred at the MSRA, MFHAS1, CLDN23, DEFB106A, DEFB105A, LOC441316, FAM90A7P and LOC441318 gene loci. Genes contained in clones are shown at the right.

and losses. The most striking finding is characterized by a high frequency of copy number losses and HDs on the short arm of chromosome 8 . Genomic changes on chromosome $8 p$ have long been considered to be one of the major drivers of 
Table I. Chromosomal recurrent minimal regions of genetic alterations on chromosome 8p in 19 stage I SCCs.

\begin{tabular}{|c|c|c|c|c|}
\hline Regions & $\begin{array}{l}\text { Gene contained } \\
\text { in clones }\end{array}$ & $\begin{array}{l}\text { 'Loss, } \\
\mathrm{n}(\%)\end{array}$ & $\begin{array}{l}{ }^{\mathrm{b}} \text { Hemizygous } \\
\text { deletion, n }(\%)\end{array}$ & $\begin{array}{l}{ }^{\mathrm{c}} \text { Homozygous } \\
\text { deletion, } \mathrm{n}(\%)\end{array}$ \\
\hline $8 \mathrm{p} 21.1-\mathrm{p} 21.3$ & $\begin{array}{l}\text { EXTL3, RC74, LOC } 340414, \text { EXTL3, } \\
\text { LOC } 389642, N K X 3-1, N K X 2-6, \\
\text { DPYSL2, DOCK5, GNRH1, KCTD9, } \\
\text { CDCA2, TNFRSF 10B, TNFRSF10C, } \\
\text { RHOBTB2, CHMP7, LOC203069, } \\
\text { LOXL2, FLJ10569, XPO7, NPM2, } \\
\text { FGF17, EPB49, RAI16, FLJ22494, } \\
\text { HR, DOK2 }\end{array}$ & $8 / 22(36.4)$ & $2 / 22(9.1)$ & $3 / 22(14.3)$ \\
\hline $8 \mathrm{p} 22$ & $\begin{array}{l}\text { LOC392206, NAT2, DLC1, TUSC } 3, \\
\text { MTUS1, LOC137012, NAT1, PDGFRL }\end{array}$ & $8 / 22(36.4)$ & $5 / 22(22.7)$ & $1 / 22(4.8)$ \\
\hline 8p23.1-p23.3 & $\begin{array}{l}\text { MFHAS1, GATA4, NEIL2, LOC 441338, } \\
\text { FDFT1, CTSB, CLDN23, MFHAS1, MSRA, } \\
\text { AGPAT5, DEFB 106A, DEFB105A, } \\
\text { LOC441316, FAM90A6P, FAM90A7P, } \\
\text { LOC441318, CSMD1, LOC157693, } \\
\text { LOC392169, FBXO25, INM01, } \\
\text { FLJ00290, LOC401441, } \\
\text { LOC389607, LOC157697, LOC } 442372\end{array}$ & $8 / 22(36.4)$ & $2 / 22(9.1)$ & $3 / 22(14.3)$ \\
\hline
\end{tabular}

${ }^{\mathrm{a} A l t e r a t i o n s}$ are defined by $\log _{2}$ ratio thresholds of -0.25 for copy number loss. ${ }^{\mathrm{b}}$ Alterations are defined by $\log _{2}$ ratio thresholds of $-0.5<\log _{2}$ ratio $<-1$ for hemizygous deletion. ${ }^{~}$ Alterations are defined by $\log _{2}$ ratio thresholds of $<-1 \log _{2}$ ratio for homozygous deletion. Using this threshold, a frequency table was generated. SCC, squamous cell carcinoma.

cancer progression, and are suspected to include critical TSGs in lung cancer (14-18). Previous investigations have focused on identifying somatic genetic mutations, including deletions and point mutations, of candidate genes on this region. Yan et al (5) reported that copy number deletions of chromosome $8 \mathrm{p}$ are one of the most prevalent genomic alterations in SCC of the lung, occurring at an incidence of 46\%, and Sy et al (6) identified a preferential association of $8 \mathrm{p}$ loss with SCC pathogenesis. Furthermore, Shao et al (19) summarized the loss of heterozygosity ( $\mathrm{LOH}$ ) of $8 \mathrm{p}$ as an early hereditary event during the development of lung cancer. Allelic losses on 8p are also well described in other carcinomas, with most studies uncovering a complex pattern that cannot be reduced to a single minimally deleted region (20). In a study by Moore et al (21), array-CGH analysis revealed a high frequency of copy number losses at $8 \mathrm{p}$ (38\%) in clear cell renal cell carcinoma, and the finding that the highest frequency of copy number alterations is on chromosome $8 \mathrm{p}$ has also described in prostate cancer (22). Notably, 8p allelic losses have also been detected in a relatively early stage during the pathogenesis of head and neck carcinomas (23). These results and the findings of the present study suggest that copy number losses on chromosome $8 \mathrm{p}$ are an important and early genetic event in the pathogenesis of lung SCC, and may harbor gatekeeper TSGs for these cancers (24).

On genomic analysis, chromosomal aberrations at the 8p21.1-p23.3 regions seem particularly noteworthy, due to the high-frequency of copy number losses and hemizygous deletions at this region, detected in 89.5 and $52.6 \%$ of the cases, respectively. Genetic alterations in the distal part of the 8p21.1-p23.3 region have been reported as early events frequently occurring in lung cancer. In addition, the size of these alterations, as well as their frequency, has also been reported to increase during lung cancer progression (25-27). These regions contain several interesting TSGs, the most attractive of which is the DCL1 gene on $8 \mathrm{p} 22$. It is considered to be one of the prime target genes on $8 \mathrm{p}$, and its reduced expression or HD has already been described in connection with various tumors, including lung cancer. Castro et al (28) reported $D L C 1$ as the most frequently methylated gene in lung tumors $(50.0 \%)$ and described the methylation of this gene as an early event, associated with early differentiation and stage. Furthermore, a significant reduction or absence of DLC1 mRNA expression has been reported in $95 \%$ of primary NSCLC, and $58 \%$ of NSCLC cell lines (29). Emerging data from Kim et al on DLC1, which encodes a RhoA GTPase-activating protein, indicate that tumor suppressor loss in NSCLC is associated with genomic deletion or epigenetic silencing and loss of $D L C 1$ gene transcription (30). Additionally, Pils et al (31) summarized the epigenetic events in a frequently deleted region on chromosome 8p22 that influences the expression of TUSC3, a putative TSG in ovarian cancer. Similarly, Vaňhara et al (32) suggested that the expression of TUSC3 epigenetically decreased in epithelial ovarian cancer, compared with that in benign controls, and provides prognostic information for patient survival. A recent study by Li et al (33) reported MTUS1 as a potential tumor suppressor in gastric cancer. The results of these studies suggest that these gene changes may 
occur as early events in the development of several different types of cancer, and may also serve as a novel prognostic indicator for lung cancer.

Previous studies have noted that other tumor suppressors associated with early cancer development are likely to exist in the 8p21.1-p23.3 regions, in addition to the handful of identified candidates. In the present study, the following possible target genes were identified at 8p23.1 in a homozygous deleted region that was previously not considered to play a pathogenic role in SCC: MSRA, MFHAS1, CLDN23, DEFB106A, DEFB105A, LOC441316, FAM90A7P and LOC441318. Although involvement of these genes in the pathogenesis of SCC has not been previously mentioned, genetic mutations of these genes have consistently been reported in multiple tumor types (31-34). Lei et al (34) reported the MSRA gene as one of the well-annotated genes significantly downregulated in hepatocellular carcinoma (HCC), and suggested that it might play a role in the progression of HCC. Furthermore, Alonso Guervós et al (17) documented an association between the loss of the MFHAS1 gene and lymph node metastases. Additionally, downregulation of the $C L D N 23$ gene in gastric cancer has also been reported $(15,16)$. These findings support the theory that genetic mutations of these developmental genes may contribute to lung tumorigenesis at an early stage, and highlight the value of examining the genomes of pre-invasive stages of cancer at tiling resolution. Moreover, the newly identified target genes at the 8p23.1 HD chromosomal sites should provide important clues with regard to the genetic mechanisms underlying the initiation and progression of stage I SCC of the lung. Further investigation is required to validate and clarify the vital functions of these genes as novel targets for early SCCs, in larger studies using multiple samples.

In the present study, the previous findings concerning the $8 p$ chromosome were significantly extended and the critical regions implicated in early SCC of the lung were firmly established. Furthermore, genomic analysis allowed the proposition of novel candidate genes that may be associated with the pathogenesis of stage I SCC cases. These findings suggest that genetic alterations on chromosome $8 \mathrm{p}$ are the first step in the initiation of genomic instability at the early onset of SCC and the newly identified target genes at the 8p23.1 HD chromosomal sites should provide important information with regard to the genetic mechanisms of the initiation and progression of stage I SCC.

\section{Acknowledgements}

This study was financially supported by the research fund of Korea Nazarene University in 2014.

\section{References}

1. Molina JR, Yang P, Cassivi SD, Schild SE and Adjei AA Non-small cell lung cancer: epidemiology, risk factors, treatment, and survivorship. Mayo Clin Proc 83: 584-594, 2008.

2. Kang JU, Koo SH, Kwon KC, Park JW and Kim JM: Gain at chromosomal region $5 \mathrm{p} 15.33$, containing TERT, is the most frequent genetic event in early stages of non-small cell lung cancer. Cancer Genet Cytogenet 182: 1-11, 2008.

3. Garnis C, Campbell J, Davies JJ, Macaulay C, Lam S and Lam WL: Involvement of multiple developmental genes on chromosome $1 \mathrm{p}$ in lung tumorigenesis. Hum Mol Genet 14: 475-482, 2005.
4. Iwakawa R, Kohno T, Kato M, Shiraishi K, Tsuta K, Noguchi M, Ogawa S and Yokota J: MYC amplification as a prognostic marker of early-stage lung adenocarcinoma identified by whole genome copy number analysis. Clin Cancer Res 17: 1481-1489, 2011.

5. Yan WS, Song LY, Wei WD, Li A, Liang QW, Liu JH and Fang Y: Chromosomal imbalance in primary lung squamous cell carcinoma and their relationship with smoking. Ai Zheng 24: 47-52, 2005 (In Chinese).

6. Sy SM, Wong N, Lee TW, Tse G, Mok TS, Fan B, Pang E, Johnson PJ and Yim A: Distinct patterns of genetic alterations in adenocarcinoma and squamous cell carcinoma of the lung. Eur J Cancer 40: 1082-1094, 2004.

7. Mihailovici MS, Danciu M, Teleman S, Stanciu C, Stan M, Bălan G and Potoroacă A: Diagnosis of gastric cancer on endobiopsies using the WHO classification. Rev Med Chir Soc Med Nat Iasi 106: 725-729, 2002 (In Romanian).

8. Hwang KT, Han W, Cho J, Lee JW, Ko E, Kim EK, Jung SY, Jeong EM, Bae JY, Kang JJ, Yang SJ, Kim SW and Noh DY: Genomic copy number alterations as predictive markers of systemic recurrence in breast cancer. Int J Cancer 123: 1807-1815, 2008.

9. Park JJ, Kang JK, Hong S, Ryu ER, Kim JI, Lee JH and Seo JS: Genome-wide combination profiling of copy number and methylation offers an approach for deciphering misregulation and development in cancer cells. Gene 407: 139-147, 2008.

10. Choi YW, Choi JS, Zheng LT, Lim YJ, Yoon HK, Kim YH, Wang YP and Lim Y: Comparative genomic hybridization array analysis and real time PCR reveals genomic alterations in squamous cell carcinomas of the lung. Lung Cancer 55: 43-51, 2007.

11. Choi YW, Bae SM, Kim YW, Lee HN, Kim YW, Park TC, Ro DY, Shin JC, Shin SJ, Seo JS and Ahn WS: Gene expression profiles in squamous cell cervical carcinoma using array-based comparative genomic hybridization analysis. Int J Gynecol Cancer 17: 687-696. 2007.

12. Kang JU, Koo SH, Kwon KC and Park JW: Frequent silence of chromosome 9p, homozygous DOCK8, DMRT1 and DMRT3 deletion at $9 \mathrm{p} 24.3$ in squamous cell carcinoma of the lung. Int J Oncol 37: 327-335, 2010.

13. Willenbrock $\mathrm{H}$ and Fridlyand J: A comparison study: applying segmentation to array CGH data for downstream analyses. Bioinformatics 21: 4084-4091, 2005.

14. Lei KF, Wang YF, Zhu XQ, et al: Identification of MSR A gene on chromosome $8 \mathrm{p}$ as a candidate metastasis suppressor for human hepatitis B virus-positive hepatocellular carcinoma. BMC Cancer 7: 172, 2007.

15. Katoh M: Epithelial-mesenchymal transition in gastric cancer (Review). Int J Oncol 27: 1677-1683, 2005.

16. Katoh $M$ and Katoh M: CLDN23 gene, frequently down-regulated in intestinal-type gastric cancer, is a novel member of CLAUDIN gene family. Int J Mol Med 11: 683-689, 2003.

17. Alonso Guervós M, Alvarez Marcos C, Llorente JL, Sampedro Nuño A, Suárez C and Hermsen M: Genetic differences between primary larynx and pharynx carcinomas and their matched lymph node metastases by multiplex ligation-dependent probe amplification. Oral Oncol 45: 600-604, 2009.

18. Kang JU, Koo SH, Kwon KC, Park JW and Kim JM: Identification of novel candidate target genes, including EPHB3, MASP1 and SST at 3q26.2-q29 in squamous cell carcinoma of the lung. BMC Cancer 9: 237, 2009.

19. Shao SJ, Wang Y and Yang PM: Abnormalities of molecular biology in premalignant lung lesions. Ai Zheng 23: 99-103, 2004.

20. Chitale D, Gong Y, Taylor BS, Broderick S, Brennan C, Somwar R, Golas B, Wang L, et al: An integrated genomic analysis of lung cancer reveals loss of DUSP4 in EGFR-mutant tumors. Oncogene 28: 2773-2783, 2009.

21. Moore LE, Jaeger E, Nickerson ML, et al: Genomic copy number alterations in clear cell renal carcinoma: associations with case characteristics and mechanisms of VHL gene inactivation. Oncogenesis 1: e14, 2012.

22. Cheng I, Levin AM, et al: Copy number alterations in prostate tumors and disease aggressiveness. Genes Chromosomes Cancer 51: 66-76, 2012.

23. El-Naggar AK, Coombes MM, Batsakis JG, Hong WK, Goepfert $\mathrm{H}$ and Kagan J: Localization of chromosome 8p regions involved in early tumorigenesis of oral and laryngeal squamous carcinoma. Oncogene 16: 2983-2987, 1998. 
24. Huang J, Zheng DL, Qin FS, Cheng N, Chen H, Wan BB, Wang YP, Xiao HS and Han ZG: Genetic and epigenetic silencing of SCARA5 may contribute to human hepatocellular carcinoma by activating FAK signaling. J Clin Invest 120: 223-241, 2010.

25. Kurimoto F, Gemma A, Hosoya Y, Seike M, Takenaka K, Uematsu K, Yoshimura A, Shibuya M and Kudoh S: Unchanged frequency of loss of heterozygosity and size of the deleted region at 8p21-23 during metastasis of lung cancer. Int J Mol Med 8: 89-93, 2001.

26. Shao S, Sun W, Zhang J, An Q, Xiao T, Yang P, Cheng S and Gao Y: Fine deletion mapping on chromosome 8p21-8p22 in adenocarcinoma of lung. Zhonghua Yi Xue Za Zhi 82: 740-742, 2002 (In Chinese)

27. Wistuba II, Behrens C, Virmani AK, et al: Allelic losses at chromosome 8 p21-23 are early and frequent events in the pathogenesis of lung cancer. Cancer Res 59: 1973-1979, 1999.

28. Castro M, Grau L, Puerta P, et al: Multiplexed methylation profiles of tumor suppressor genes and clinical outcome in lung cancer. J Transl Med 8: 86, 2010.

29. Yuan BZ, Jefferson AM, et al: DLC-1 operates as a tumor suppressor gene in human non-small cell lung carcinomas. Oncogene 23: 1405-1411, 2004.
30. Kim TY, Jackson S, Xiong Y, Whitsett TG, Lobello JR, Weiss GJ, Tran NL, Bang YJ and Der CJ: CRL4A-FBXW5-mediated degradation of DLC1 Rho GTPase-activating protein tumor suppressor promotes non-small cell lung cancer cell growth. Proc Natl Acad Sci USA 110: 16868-16873, 2013.

31. Pils D, Horak P, Vanhara P, Anees M, Petz M, Alfanz A, Gugerell A, Wittinger M, Gleiss A, Auner V, Tong D, Zeillinger R, Braicu EI, Sehouli J and Krainer M: Methylation status of TUSC3 is a prognostic factor in ovarian cancer. Cancer 119: 946-954, 2013.

32. Vaňhara P, Horak P, Pils D, Anees M, Petz M, Gregor W, Zeillinger R and Krainer M: Loss of the oligosaccharyl transferase subunit TUSC3 promotes proliferation and migration of ovarian cancer cells. Int J Oncol 1383-1389, 2013.

33. Li X, Liu H, Yu T, Dong Z, Tang L and Sun X: Loss of MTUS1 in gastric cancer promotes tumor growth and metastasis. Neoplasma 61: 128-135, 2014.

34. Lei KF, Wang YF, Zhu XQ, Lu PC, Sun BS, Jia HL, Ren N, Ye QH, Sun HC, Wang L, Tang ZY and Qin LX: Identification of MSRA gene on chromosome $8 p$ as a candidate metastasis suppressor for human hepatitis B virus-positive hepatocellular carcinoma. BMC Cancer 7: 172, 2007. 